\title{
Prevalencia de desnutrición en menores de cinco años de Tabasco
}

\author{
Lucio G. Lastra-Escudero, M.C., (1) Silvia G. Roldán-Fernández, M.C., M.S.P., (2) \\ Enrique Hernández-Martínez, M.C., M.S.P., ${ }^{(3)}$ Alma Patricia Hernández-Torres, Lic. N utr., M.S.P., M. en C., ${ }^{(4)}$ \\ Félix Gerardo Lechuga-Padrón, M.C., M.S.P. ${ }^{(5)}$
}

\section{Lastra-Escudero LG, Roldán-Fernández SG, Hernández-Martínez E, Hernández-Torres AP, Lechuga-Padrón FG. \\ Prevalencia de desnutrición en menores de cinco años de Tabasco. Salud Publica Mex 1998;40:408-414.}

\section{Resumen}

Objetivo. Evaluar la prevalencia de desnutricion que se presentó en niños tabasqueños menores de cinco años en 1996, y su evolución entre 1991 y 1996. Material y métodos. Se realizó un estudio de corte transversal que incluyó a 1256 niños de 593 comunidades (31 urbanas y 562 rurales) de los 17 municipios de Tabasco. Resultados. El indicador peso/edad registró $41 \%$ de prevalencia de desnutrición, distribuida en leve (26.12\%), moderada (12.62\%) y severa (2.39\%). En niños de entre 1 y 5 años la prevalencia de desnutrición fue de 45\%. Conclusiones. D e 1991 a 1996 la cifra de niños con desnutrición ha disminuido proporcionalmente al incremento de la nutrición normal. Asimismo, han disminuido de seis a cuatro los municipios con indicadores críticos de desnutrición, con base en las acciones del sector salud en el estado de Tabasco.

Palabras clave: trastornos de la nutrición infantil/prevalencia; antropometría; México

\author{
Lastra-Escudero LG, Roldán-Fernández SG, \\ Hernández-Martínez E, Hernández-Torres AP, \\ Lechuga-Padrón FG. \\ Malnutrition prevalence in children \\ under 5 years old in Tabasco, Mexico. \\ Salud Publica Mex 1998;40:408-414.
}

\begin{abstract}
A bstract
Objective. To evaluate malnutrition prevalence in children under five years-old in Tabasco, Mexico, during 1996, and their evolution in 1991-1996. Material and methods. A cross-sectional study, including 1256 children under five years-old in 593 communities (31 urban and 562 rural) from 17 sanitary districts. Results. The weight/age indicator for malnutrition in children under five years old showed $59 \%$ of children had normal nutrition and $41 \%$ with malnutrition, distributed as follows: $26.12 \%$ slight, $12.62 \%$ moderate and $2.39 \%$ severe. In children between $1-5$ years old, malnutrition prevalence was $45 \%$. Conclusions. The number of children with malnutrition in Tabasco during the study period (1991-1996) has decreased in proportion to the increase of those with normal nutrition. Based on the health system activities, the number of health districts with critical malnutrition indicators decreased from 6 to 4 .
\end{abstract}

Key words: infant nutrition disorders/prevalence; anthropometry; Mexico
U ace cuarenta años se inició formalmente en Méxi- co el estudio de la nutrición y la alimentación. ${ }^{1-3}$ El Instituto Nacional de la Nutrición Salvador Zubirán (INNSZ) ha realizado 49 encuestas, entre las que destaca la Encuesta Nacional de Nutrición y Alimentación aplicada en los años de 1974, 1979, ${ }^{4} 1989^{5}$ y $1996 .{ }^{6}$ En la década de los años ochenta se produjo un vacío casi absoluto de información en cuanto a la vigilancia

(1) Secretario de Salud del Gobierno del Estado de Tabasco, México.

(2) Directora de Programas Preventivos, Secretaría de Salud del Gobierno del Estado de Tabasco (SSA-Tabasco), México.

(3) Secretario Técnico de la SSA-Tabasco, México.

(4) Jefe del Departamento de Proyectos e Investigación, SSA-Tabasco, México

(5) Jefe del Departamento de Promoción de la Salud, SSA-Tabasco, México

Fecha de recibido: 26 de febrero de 1998 - Fecha de aprobado: 15 de julio de 1998 Solicitud de sobretiros: Dr. Enrique Hernández Martínez. Secretaría de Salud de Tabasco, Centro Administrativo de Gobierno. Av. Paseo Tabasco 1504, Tabasco 2000, 86035 Villahermosa, Tabasco, México. 
epidemiológica de la nutrición, hasta el año de 1988, cuando la Dirección General de Epidemiología llevó a cabo la Encuesta Nacional de Nutrición. ${ }^{7}$ Además de esas encuestas, el Sistema para el Desarrollo Integral de la Familia realizó en 1993 y 1994, 8,9 el Censo Nacional de Talla en Escolares.

En forma paralela a esos estudios, en México se han realizado diversos programas con el propósito de mejorar las condiciones de nutrición. ${ }^{10}$ Desafortunadamente la tradición en la investigación nutricional con cobertura nacional ha tenido como limitante la desvinculación con las instancias operativas, debido sobre todo a que la publicación de los estudios se ha hecho por regiones y, en pocos casos, por estado. Con esos resultados no ha sido posible retroalimentar a las jurisdicciones sanitarias y/o los municipios, instancias en donde se programan las acciones orientadas a la población. Baste señalar que en los últimos cuarenta años no ha habido un programa de atención a la desnutrición de alcance nacional en el medio rural que se sustente en los resultados de esas encuestas.

En este marco, en 1991 la Secretaría de Salud del Gobierno del estado de Tabasco, ${ }^{11}$ con asesoría del INNSZ y del Instituto Nacional de Salud Pública (INSP), realizó la I Encuesta Estatal de Nutrición, cuyos resultados representan para el estado la aproximación más confiable a las condiciones de nutrición del prescolar tabasqueño. De acuerdo con sus resultados, se han llevado a cabo intervenciones institucionales que han fortalecido al Programa Estatal de Nutrición, con la creación de la Fundación para la Nutrición en Tabasco (Pronutra), que comenzó en 1993 realizando acciones de detección-atención en los municipios con las prevalencias de desnutrición más altas (Macuspana y Tacotalpa); asimismo, en 1994 se creó el Centro Infantil para la Nutrición de Tabasco (CINTA), entre otras acciones.

Sin embargo, ante la transición epidemiológica, demográfica y de los sistemas de salud ${ }^{12}$ y frente al cambio en la tendencia histórica de indicadores básicos de salud, ${ }^{*}$ después de cinco años los resultados perdieron vigencia para la planeación de acciones y para sustentar la toma de decisiones en el abordaje del problema nutricional dentro del estado, por lo que fue necesario actualizar la información y, al mismo tiempo, evaluar los cambios en los niveles de desnutrición infantil entre 1991 y 1996.

\footnotetext{
* Tabasco Salud 2000. Secretaría de Salud del Gobierno del Estado de Tabasco, 1997. Documento no publicado.
}

Con este propósito la Secretaría de Salud del Gobierno del Estado de Tabasco llevó a cabo, entre agosto y octubre de 1996, ${ }^{*}$ la II Encuesta Estatal de Nutrición 1996 utilizando la misma metodología de 1991 para facilitar comparaciones. La encuesta de 1996 consideró como unidad de análisis a los niños de 1 a 5 años de edad y, a diferencia de la hecha en 1991, incluyó también a los menores de un año; la muestra fue seleccionada probabilísticamente.

Este estudio es el primer producto de una serie que se elaborará a partir de los resultados de la II Encuesta Estatal de Nutrición 1996, por lo que se describen fundamentalmente las prevalencias de la desnutrición obtenidas en los tabasqueños menores de cinco años, así como la frecuencia y la distribución de la desnutrición de acuerdo con la edad, el sexo y el área de residencia (urbana y rural). De igual manera se muestra el cambio ocurrido entre 1991 y 1996, por medio de la comparación de las prevalencias que se presentaron en los niños de entre 12 y 60 meses de edad, en ambos años, mediante el indicador peso para la edad.

\section{Material y métodos}

En las encuestas transversales realizadas en 1991 y 1996 se utilizaron muestras probabilísticas, calculadas con un nivel de confianza de 95\%, una precisión de 3\% y un poder estadístico de $80 \%$. La prevalencia teórica de desnutrición esperada para 1991 fue de 30\%, y para 1996, de $46 \%$.11,*

El tamaño de la muestra en 1991 fue de 935 niños de entre 12 y 60 meses de edad, y en 1996 fue de 1252 niños de entre 0 y 60 meses. El universo de estudio fueron los niños menores de cinco años. Ambas encuestas cubrieron las áreas urbana y rural. En 1991 se incluyeron 509 localidades (18 urbanas y 491 rurales), ${ }^{11}$ y en 1996, 593 (31 urbanas y 562 rurales).

El muestreo para la encuesta de 1996 fue polietápico; así, en una primera etapa se determinó el tamaño de la muestra estatal en unidades de estudio (prescolares). Después se realizó la asignación proporcional al tamaño de cada jurisdicción sanitaria, que en el caso de Tabasco corresponde a cada municipio (se conformaron 17 estratos). Finalmente, se seleccionaron sistemáticamente los niños de cada municipio con base en el Registro Nominal del Programa de Vacunación,

\footnotetext{
* Protocolo de la II Encuesta Estatal de Nutrición. Secretaría de Salud del Gobierno del Estado de Tabasco, 1996. Documento no publicado.
} 
que en Tabasco tiene una congruencia de $97.8 \%$ con la población de menores de cinco años proyectada por el Instituto Nacional de Estadística, Geografía e Informática. ${ }^{*}, 13$

El cuestionario que se aplicó en la II Encuesta Estatal de Nutrición 1996 fue el mismo que se utilizó en la Encuesta Nacional de Alimentación del Medio Rural 1996 (ENAL96). ${ }^{6}$ Antes del levantamiento el INNSZ aplicó una prueba piloto en el estado de Morelos. La capacitación a las encuestadoras y la estandarización estuvieron a cargo del personal del INNSZ, que recurrió a la metodología propuesta por Habitch. ${ }^{14}$ Para hacer las mediciones antropométricas se utilizaron infantómetros Dynatop con precisión de $0.5 \mathrm{~cm}$, estadímetros de escuadra y un flexómetro Dynatop con precisión de $1 \mathrm{~cm}$, así como básculas tipo Salter de $20 \mathrm{~kg}$ de capacidad, con una precisión de $100 \mathrm{~g}$. Personal normativo de la Oficina de Nutrición de la Secretaría de Salud del Gobierno del Estado de Tabasco se encargó del procesamiento electrónico de la información, de la supervisión de la encuesta, así como de la captura y la clasificación de los datos.

La clasificación del estado de nutrición se basó en la estimación probabilística mediante unidades de desviación estándar (puntuación $z$ ) de la relación del peso con la edad, de acuerdo con la población de referencia del National Center for Health Statistics-Organización Mundial de la Salud (NCHS-OMS) $\cdot{ }^{15}$ En esa clasificación la condición de normal se asume cuando el peso medido es menor a $2 z$, y mayor o igual a $-1 z$. La desnutrición se establece en tres rangos por debajo del valor del peso correspondiente a -1z: leve, menor de $-1 z$ y mayor de $-2 z$; moderada, menor o igual a $-2 z$ y mayor que $-3 z$, y severa, cuando el peso observado corresponde a menos de $-3 z$.

La información se procesó en el INNSZ utilizando el programa SPSS, versión 6.1 / Windows 95, y en el análisis de la información antropométrica se utilizó el programa denominado Sistema de Cálculo Antropométrico (SCAIN), que efectúa la valoración antropométrica con base en la puntuación $\mathrm{z}$ bajo la referencia NCHS-OMS, y que fue diseñado en la Subdirección de Nutrición de la Comunidad, del INNSZ. El intervalo de edad de los niños participantes fue de 0 a 60 meses; el SCAIN los seleccionó automáticamente, estableciendo un punto de corte entre la fecha de nacimiento y la fecha de la entrevista.

\footnotetext{
* Sistema de registro y control de vacunación en menores de cinco años (PROVAC). Secretaría de Salud del Gobierno del Estado de Tabasco, 1996. Documento no publicado.
}

En la estimación de la prevalencia general de desnutrición se ponderó el número de niños, de entre 12 y 60 meses de edad, cuyo peso se encontró, dentro del rango de las puntuaciones $z$, por debajo del valor del peso correspondiente a -1 desviación estándar (DE) del porcentaje teórico esperado. Además, se obtuvo la proporción de desnutrición en los niños que conformaron los siguientes grupos de edad: a) menores de 1 año (<12 meses); b) de 1 a 4 años ( $>12$ a 48 meses), y c) mayores de 4 a 5 años ( $>48$ a 60 meses). Se consideró esta distribución por grupos de edad debido a que es similar a la de los grupos blanco de las acciones del Programa Estatal de Nutrición.

En ambas encuestas el estado de nutrición, que puede clasificarse en nutrición normal y desnutrición en sus tres grados (indicador peso/edad), se obtuvo mediante frecuencias simples y medidas de tendencia central (medias), con intervalos de confianza (IC) de 95\%. La relación del estado de nutrición con la edad, el sexo y el área de residencia (urbana y rural) se obtuvo mediante un análisis bivariado.

Para observar el cambio en la prevalencia general de desnutrición entre 1991 y $1996,{ }^{16}$ se consideró la distribución del indicador peso/edad de acuerdo con el número de niños por debajo de la mediana -1 DE y con el número de niños por debajo de la mediana -2 DE. La proporción prevista de disminución de la desnutrición fue de $1.6 \%$ por debajo de la mediana -1 DE y de $0.68 \%$ por debajo de la mediana -2 DE. Para estimarla se consideraron las prevalencias de desnutrición de la región Golfo Sureste de la República mexicana, notificadas con base en el indicador peso para la edad en las encuestas nacionales del INNSZ, correspondientes a 1974, 1979, 1989 y 1996, pues se carece de datos precisos sobre años anteriores que refieran particularmente las prevalencias de la desnutrición en el estado de Tabasco; la información que se ha publicado se refiere exclusivamente al estado de nutrición por zonas. La prueba estadística del cambio en las prevalencias de desnutrición se realizó mediante la comparación de dos proporciones muestrales independientes -con la prueba de $z-$, y la desnutrición por estratos (leve, moderada y severa) se comparó mediante la prueba de $\chi^{2}$.

\section{Resultados}

En el cuadro I se observa que en los 1252 niños tabasqueños de entre 0 y 60 meses de edad que se estudiaron en 1996, la prevalencia global de desnutrición fue de $41 \%$ con un IC 95\% de 40.7-48.4. De ese grupo, 26.12\% tenía desnutrición leve, con -1 DE por debajo de la mediana, y $15.01 \%$ presentó alto riesgo nutricional, con -2 


\section{Cuadro I

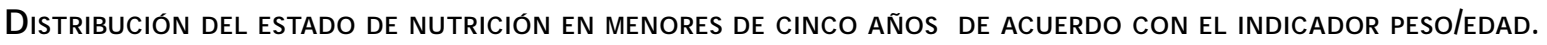 Tabasco, México, 1996}

\begin{tabular}{|c|c|c|c|c|c|c|c|}
\hline \multirow[b]{2}{*}{ M unicipios } & \multirow{2}{*}{$\begin{array}{c}\text { Número de niños } \\
\text { estudiados } \\
n\end{array}$} & \multirow[b]{2}{*}{$\begin{array}{c}\text { Normales } \\
\%\end{array}$} & \multicolumn{5}{|c|}{ Desnutridos } \\
\hline & & & $\begin{array}{c}\text { Totales } \\
\%\end{array}$ & $\begin{array}{l}\text { Leves } \\
\%\end{array}$ & $\begin{array}{l}\text { Moderados } \\
\%\end{array}$ & $\begin{array}{l}\text { Severos } \\
\%\end{array}$ & $\begin{array}{c}\text { Alto riesgo moderados y severos } \\
\%\end{array}$ \\
\hline Balancán & 56 & 70 & 30 & 14.29 & 14.30 & 1.8 & 16.10 \\
\hline Cárdenas & 147 & 54 & 46 & 26.53 & 17.69 & 1.4 & 19.00 \\
\hline Centla & 89 & 45 & 55 & 37.08 & 16.85 & 1.1 & 18.00 \\
\hline Centro & 141 & 75 & 25 & 17.02 & 6.38 & 1.4 & 8.00 \\
\hline Comalcalco & 103 & 66 & 34 & 22.33 & 10.68 & 1.0 & 11.68 \\
\hline Cunduacán & 88 & 64 & 36 & 20.45 & 8.00 & 8.0 & 16.00 \\
\hline E. Zapata & 20 & 65 & 35 & 30.00 & 5.00 & 0.0 & 5.00 \\
\hline Huimanguillo & 118 & 48 & 52 & 33.90 & 14.41 & 1.7 & 16.10 \\
\hline Jalapa & 27 & 78 & 22 & 22.22 & 0.00 & 0.0 & 0.00 \\
\hline Jalpa de Méndez & 62 & 47 & 53 & 37.09 & 12.90 & 3.2 & 16.13 \\
\hline Jonuta & 24 & 58 & 42 & 12.50 & 29.16 & 0.0 & 29.16 \\
\hline Macuspana & 107 & 50 & 50 & 25.23 & 21.00 & 4.0 & 24.30 \\
\hline $\mathrm{N}$ acajuca & 66 & 59 & 41 & 21.21 & 15.15 & 4.5 & 19.70 \\
\hline Paraíso & 60 & 58 & 42 & 30.00 & 8.33 & 3.3 & 12.00 \\
\hline Tacotalpa & 57 & 56 & 44 & 35.09 & 7.00 & 1.8 & 9.00 \\
\hline Teapa & 29 & 69 & 31 & 31.03 & 0.00 & 0.0 & 0.00 \\
\hline Tenosique & 58 & 59 & 41 & 24.14 & 13.80 & 3.4 & 17.24 \\
\hline Total & 1252 & 59 & 41 & 26.12 & 12.62 & 2.39 & 15.01 \\
\hline
\end{tabular}

N ota: clasificación del estado de nutrición, con base en la puntuación z. N ational Center for Health Statistics-O rganización Mundial de la Salud (N CHSOMS)

Fuente: Encuesta Estatal de N utrición 1996

DE por debajo de la mediana. Este grupo incluyó a los desnutridos moderados (12.62\%) y a los severos (2.39\%).

En la figura 1 se muestran las prevalencias de desnutrición desagregadas por subgrupos de edad; éstas fueron de $25.3 \%$ para los niños de entre 0 y 12 meses; de $43.7 \%$ para los de $>12$ a 48 meses, y de $48.5 \%$ para los de $>48$ a 60 meses.

El problema de bajo peso para la edad fue mayor en el área rural que en la urbana (figura 2). Por otra parte, en la distribución del estado de nutrición por sexo se hizo evidente una ganancia en el rango de normalidad de peso para la edad en el grupo de niñas participantes en el estudio (51.3\%), en comparación con el grupo de niños $(48.7 \%)$.

Asimismo, en las encuestas estatales (1991 y 1996) se registró una proporción mayor de niños con peso normal para la edad en relación con la proporción de niños desnutridos.

En el cuadro II se muestra que la prevalencia de desnutrición que se encontró en 1991 en niños de 12 a 60 meses de edad fue de $46 \%$, distribuida de la si- guiente manera: $29.8 \%$ correspondió a la desnutrición leve (por debajo de la mediana $-1 \mathrm{DE}$ ), y $16.2 \%$, a la moderada y severa (por debajo de la mediana $-2 \mathrm{DE}$ ). Asimismo, se aprecia la prevalencia de desnutrición de 1996, que fue de 44.9\% con un IC 95\% de 41.8-48.2; $28.7 \%$ correspondió a la desnutrición leve (por debajo de la mediana $-1 \mathrm{DE})$, y $16.2 \%$, a la moderada y severa (por debajo de la mediana -2 DE).

Al comparar las prevalencias de desnutrición de 1991 y 1996 a partir de las dos encuestas estatales, se consideró el porcentaje previsto de disminución de la desnutrición, que fue de $1.60 \%$ para la desnutrición leve y de $0.68 \%$ para la moderada y severa. Como resultado se observó que, a cinco años de diferencia, la desnutrición leve disminuyó $1.1 \%$ por debajo de la mediana $-1 \mathrm{DE}$, y la moderada y severa disminuyó $0.1 \%$ por debajo de la mediana $-2 \mathrm{DE}$. El cambio en el estado nutricional fue estadísticamente significativo $(p<$ 0.0001 ), a partir de la comparación de dos proporciones muestrales independientes -utilizando las prevalencias de la denutrición de 1991 y 1996-, y con la prueba de 


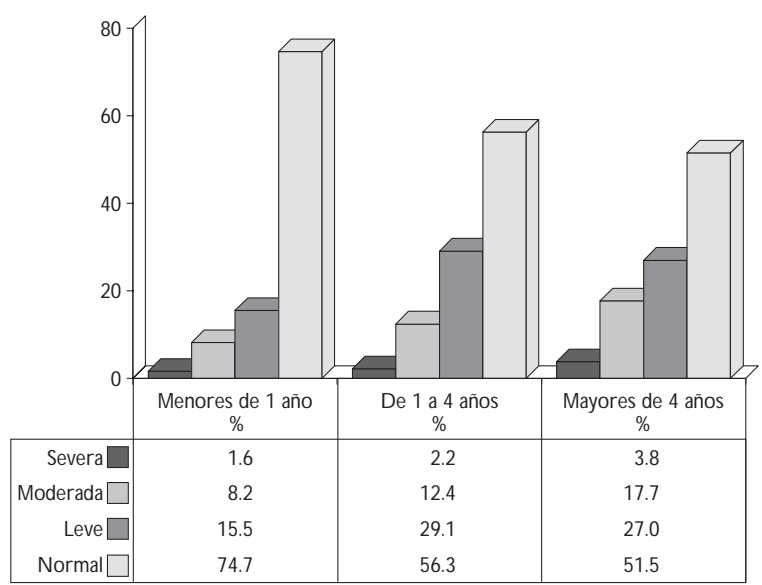

N ota: clasificación del estado de nutrición, con base en la puntuación z. N CHS-OMS

Fuente: Encuesta Estatal de N utrición 1996

Figura 1. Distribución por grupos de edad del estaDO DE NUTRICIÓN DE LOS NIÑOS MENORES DE CINCO AÑOS, DE ACUERDO CON EL INDICADOR PESO/EDAD.TABASCO, MÉxıco, 1996

$\chi^{2}$ de acuerdo con el valor observado y el valor previsto de la desnutrición.

En la figura 3 se presenta un mapa nutricional que considera a los 17 municipios del estado de Tabasco e indica la localización de las prevalencias de desnutrición moderada y severa (desnutrición de alto riesgo). Si bien el tamaño de la muestra impide hacer inferencias confiables en cuanto a los municipios, se muestra este mapa debido a su utilidad práctica y a la importancia de apreciar el problema en el nivel jurisdiccional, en el sentido de que la focalización de los

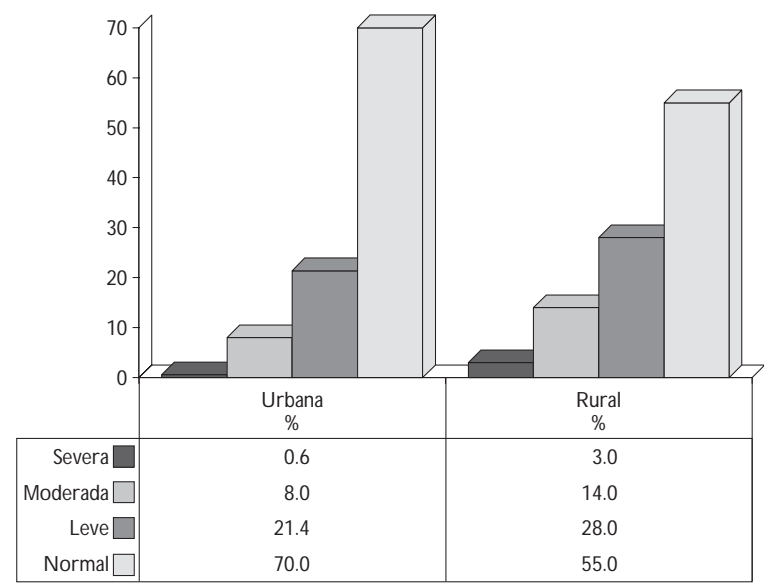

N ota: clasificación del estado de nutrición, con base en la puntuación z. N CHS-O MS

Fuente: Encuesta Estatal de N utrición 1996

Figura 2. Distribución del estado de nUtrición de LOS NIÑOS MENORES DE CINCO AÑOS, SEGÚN EL ÁREA DE RESIDENCIA URBANA O RURAL, DE ACUERDO CON EL INDICADOR PESO/EDAD.TABasco, MÉxico, 1996

niños desnutridos en los municipios ha constituido una ventaja para la planeación y la distribución de los recursos.

La Encuesta Estatal de Nutrición de 1996 permitió observar que había cuatro municipios con prevalencia de desnutrición mayor a 20\%, dos menos que en la encuesta de 1991. De acuerdo con esa información, los municipios que continúan afectados con las prevalencias más altas de desnutrición (moderada y severa) son Macuspana, Jonuta, Centla y Nacajuca; actualmente se les considera como áreas críticas y priorita-

\section{Cuadro II}

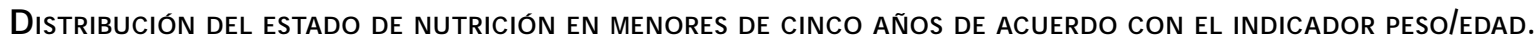
Tabasco, Méxıco, 1991 y 1996

Estado de nutrición

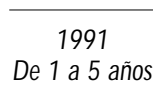

$\%$
Encuestas estatales de nutrición

\begin{tabular}{cc}
\multicolumn{3}{c}{1996} \\
\hline $\begin{array}{c}\text { De } 1 \text { a } 5 \text { años } \\
\%\end{array}$ & De 0 a 5 años \\
&
\end{tabular}

Encuesta Nacional de Alimentación y Nutrición en el M edio Rural 1996 De 0 a 5 años $\%$

\begin{tabular}{lcccc} 
N utrición normal & 54 & 55.1 & 59 & 54.6 \\
\hline Desnutrición & 46 & 44.9 & 41 & 45.4 \\
\hline Leve & 29.8 & 28.7 & 26.12 & 30.4 \\
\hline Moderada & 12.8 & 13.6 & 12.62 & 12.1 \\
\hline Severa & 3.4 & 2.6 & 2.39 & 2.9
\end{tabular}

N ota: clasificación del estado de nutrición, con base en la puntuación z. N CHS-O MS

Fuente: encuestas estatales de nutrición 1991 y 1996 

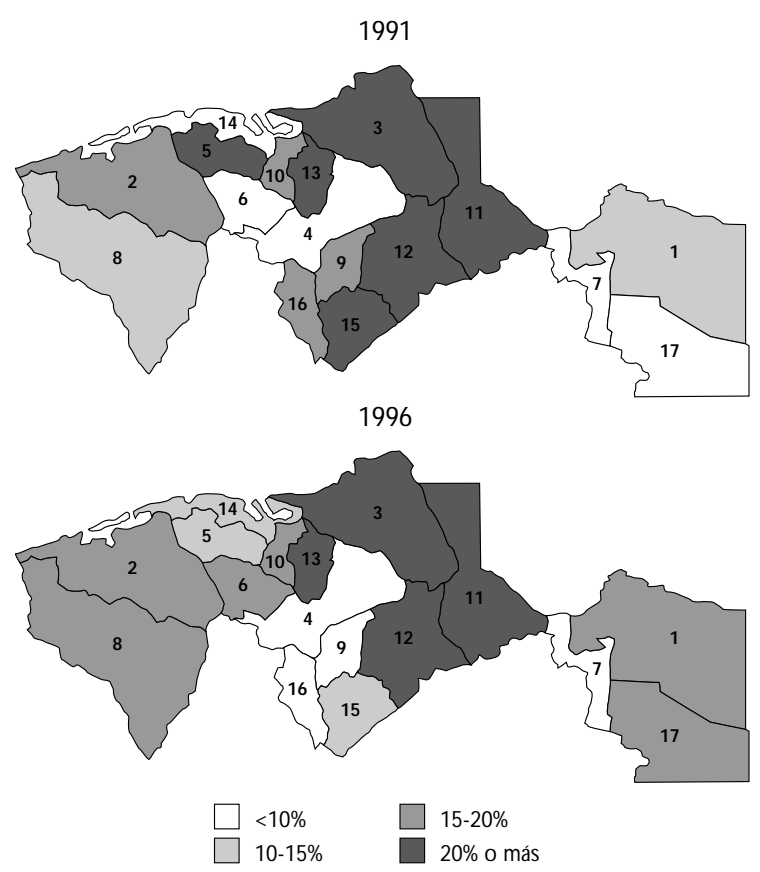

$\begin{array}{lll}\text { 1. Balancán } & \text { 7. E. Zapata } & \text { 13. Nacajuca } \\ \text { 2. Cárdenas } & \text { 8. Huimanguillo } & \text { 14. Paraíso } \\ \text { 3. Centla } & \text { 9. Jalapa } & \text { 15.Tacotalpa } \\ \text { 4. Centro } & \text { 10. Jalpa de Méndez } & \text { 16.Teapa } \\ \text { 5. Comalcalco } & \text { 11. Jonuta } & \text { 17.Tenosique } \\ \text { 6. Cunduacán } & \text { 12. Macuspana } & \end{array}$

Fuente: encuestas nacionales de nutrición 1991 y 1996

Figura 3. Prevalencia de desnutrición de alto riesGO (MODERADA Y SEVERA) EN NIÑOS DE 1 A 5 AÑOS, DE ACUERDO CON EL INDICADOR PESO/EDAD.TABASCO, MÉXICO, 1991 Y 1996

rias para la planeación y la distribución de recursos en materia de nutrición. Por otra parte, en ambas encuestas los municipios Centro y Emiliano Zapata tuvieron prevalencias de desnutrición menores a $10 \%$.

\section{Discusión}

Los resultados de la II Encuesta Estatal de Nutrición ponen de manifiesto que la desnutrición en Tabasco sigue siendo un problema de salud pública en ese estado de la República mexicana. La prevalencia de desnutrición de $41 \%$ en los niños menores de cinco años muestra que aproximadamente $105371^{*}$ niños padecen desnutrición; esa cifra se traduce en demandas de

\footnotetext{
* Proyecciones de población 1997. Dirección de Planeación, Secretaría de Salud del Gobierno del Estado de Tabasco, 1997. Documento no publicado.
}

atención nutricional que deben dirigirse a 66820 niños desnutridos leves, 33410 moderados y 5140 con desnutrición severa. Todos requieren de atención especializada y alimentación complementaria para poder subsistir, sobre todo en los primeros años de vida. ${ }^{17}$

Los niños desnutridos habitan, sobre todo, en el área rural del estado. Las prevalencias más altas se registraron en los municipios de Centla, Jonuta, Nacajuca, Macuspana, y Tenosique, que son municipios con índices de ruralidad de 95 a $99.5 \% .^{18}$ Además, en esos municipios se ubica la mayor proporción de población indígena del estado.

Las prevalencias de desnutrición leve notificadas pueden atribuirse, en particular, a las malas condiciones higiénicas y dietéticas que predominan en el hogar, mismas que Pelletier, ${ }^{19}$ en sus estudios epidemiológicos de nutrición, ha señalado como potenciadores de la desnutrición en los países en vías de desarrollo, afirmando que la desnutrición leve y moderada es la responsable de más de $80 \%$ de las defunciones infantiles.

A la luz del análisis de la II Encuesta Estatal de Nutrición 1996 y de las prevalencias de desnutrición de los grupos etáreos constituidos de acuerdo con las necesidades del Programa Estatal de Nutrición, se observó que el grupo de prescolares (>de 12 a 60 meses) es el más afectado, pues presenta una prevalencia ponderada de $46.1 \%$, en relación con la prevalencia de desnutrición de $25.3 \%$ en el grupo de menores de un año (de 0 a <de 12 meses); la razón fue de 1.8: 1, lo cual destaca que el riesgo es mayor en los niños prescolares. Puede deducirse que estos niños en general provienen de familias de escasos recursos y con limitado acceso a los servicios de salud, o que probablemente son rehabilitados en los centros de atención y que reinciden en la desnutrición al llegar a sus hogares. Ante esta situación se ha propuesto la medida de reforzar la atención a los prescolares desnutridos en el estado.

En forma paralela a la II Encuesta Estatal de Nutrición, en 1996 se llevó a cabo la Encuesta Nacional de Alimentación y Nutrición en el Medio Rural (ENAL 96), ${ }^{6}$ cuyos resultados mostraron que en Tabasco la prevalencia de desnutrición en los menores de cinco años fue de $45 \%$, es decir, que se registraron 4.4 puntos porcentuales más de desnutrición que en la II Encuesta Estatal de Nutrición 1996 (cuadro II). La diferencia puede atribuirse a que esta última se aplicó en las áreas urbana y rural, y que los niños desnutridos se localizan principalmente en las comunidades rurales.

La disminución del índice de desnutrición entre 1991 y 1996 puede atribuirse a las intervenciones institucionales que se generaron como resultados de la 
I Encuesta Estatal de Nutrición, en particular al fortalecimiento del Programa Estatal de Nutrición, que ha dirigido sus acciones a los municipios con las prevalencias más altas de desnutrición en 1991, y a las acciones desarrolladas por Pronutra. Además, las jurisdicciones sanitarias han canalizado a los niños con disminución moderada y severa detectados en sus unidades al CINTA, y se ha atendido de manera simultánea el Programa de Capacitación a las Madres de Niños Desnutridos, ${ }^{20,21}$ considerando básicamente la reorientación de los hábitos alimentarios y sanitarios de las familias.

\section{Conclusiones}

La II Encuesta Estatal de Nutrición 1996 muestra que entre 1991 y 1996 ha disminuido la desnutrición en 1.2 puntos porcentuales y que, consecuentemente, se ha incrementado el porcentaje de niños con nutrición normal de acuerdo con el indicador de peso para la edad.

En 1996 se presentó un balance entre la disminución del grado de desnutrición severa y el incremento del grado de desnutrición moderada; es decir, en Tabasco existen menos niños con desnutrición severa que hace cinco años, lo que sugiere cambios discretos pero importantes, acordes con las tendencias esperadas para una modificación sustancial del estado de nutrición en la población infantil.

\section{Agradecimientos}

Las encuestas estatales de nutrición en Tabasco se llevaron a cabo gracias al invaluable apoyo del doctor Abelardo Avila Curiel y de la licenciada Teresa Shamah Levy del INNSZ. Por otra parte, se agradece la colaboración del personal del Departamento de Promoción de la Salud de la Secretaría de Salud del Gobierno del Estado de Tabasco, en particular de la licenciada Dinorah Méndez Sosa y del equipo de encuestadoras y supervisores que coordinaron la encuesta.

\section{Referencias}

1. Chávez-Villasana A. Perspectivas de la nutrición en México. México, D.F.: Instituto N acional de la N utrición Salvador Zubirán, 1982;L-50:1-18.
2. Roldán A, C hávez-Villasana A, Romero G, Madrigal H, Peláez M. Geografía del hambre en México. Redefinición de zonas críticas en nutrición. México, D.F.: Instituto N acional de la Nutrición Salvador Zubirán, 1988; L-79:1-46.

3. Chavéz A. Encuestas nutricionales en México. 2a. edición. México, D.F.: División de Nutrición de Comunidad, Instituto $\mathrm{N}$ acional de la N utrición Salvador Zubirán, 1974;L-1:2.

4. Madrigal H, Moreno O, Chávez A. Encuesta N acional de Alimentación 1979. Resultados de la encuesta rural por entidad federativa y desagregada según zonas nutricionales. México, D.F.: División de N utrición, Instituto $\mathrm{N}$ acional de la Nutrición Salvador Zubirán, 1982.

5. Instituto $\mathrm{N}$ acional de la N utrición Salvador Zubirán. Encuesta $\mathrm{N}$ acional deAlimentación en el Medio Rural. EN AL-89. México D.F.: Instituto N acional de la N utrición Salvador Zubirán/C omisión N acional deAlimentación, 1990.

6. Avila-C uriel A, Shamah-Levy T, Chavéz-Villasana A. Encuesta N acional deA limentación y $N$ utrición en el Medio Rural, 1996. México, D.F.:Instituto N acional de la N utrición Salvador Zubirán, 1997:1-93.

7. Dirección General de Epidemiología, Secretaría de Salud. Encuesta Nacional de N utrición 1988. Resultados nacionales y por regiones. México, D.F.: DGE, SSA, 1988.

8. Sistema Nacional para el Desarrollo Integral de la Familia. I Censo $\mathrm{N}$ acional de Talla. México D.F.: DIF/SEP, 1994.

9. Sistema Nacional para el Desarrollo Integral de la Familia. II Censo N acional de Talla. México D.F.: DIF/SEP, 1997.

10. Rivera J, Long K, González-Cossío T, Parra S, Rivera M, Rosado JL. Nutrición y salud. México D.F.: Secretaría de Salud, Cuadernos de Salud, 1994.

11. Hernández-Martínez E, Roldán-Fernández S. Prevalencia de desnutrición en prescolares de Tabasco. Salud Publica Mex 1995;37:211-218.

12. Ruelas BE. Calidad, productividad y costos. Salud Publica Mex 1993; 35:298-303.

13. Instituto Nacional de Estadística, Geografía e Informática. Anuario estadístico del estado de Tabasco. México, D.F.: IN EGI, 1995.

14. Habitch J P. Estandarización de métodos epidemiológicos cuantitativos sobre el terreno. Bol 0 ficina Sanit Panam 1974;76:375-384.

15. Michael J, D ibley MJ, Stahling N , Phillip N , Frederick L, Trowbridge FL. Interpretation of z-score anthropometric indicators derived from the international growth reference. Am J C lin N utr 1987;46:749-762.

16. World Health 0 rganization. Measuring chance in nutritional status. Ginebra: W HO, 1983.

17. Monkeberg F. D esnutrición infantil. Santiago de C hile: Impresora C reces, 1988:2-4.

18. Instituto Nacional de Estadística, Geografía e Infomática, Conteo de población y vivienda 1995, Resultados definitivos: tabulados básicos. México, D.F.: IN EGI, 1995.

19. Pelletier D, Frongill E. Efectos de la malnutrición en la mortalidad de menores de 5 años en países en desarrollo. Bol 0 ficina Sanit Panam 1996;120(5):425-431

20. Lastra-Escudero L, Hernández-Martínez E. Desnutrición infantil en Tabasco, un problema prioritario de salud pública. Salud de Tabasco 1996; 2:152-156.

21. Secretaría de Salud del Gobierno del Estado de Tabasco. Modelo de atención a la salud a población abierta.Villahermosa: SSA-Tabasco, 1996. 